\title{
Energetic Approach to Large Strain Gradient Crystal Plasticity
}

\author{
Jan Kratochvíl ${ }^{1}$, Martin Kružík $k^{2,1}$ \\ ${ }^{1}$ Czech Technical University, Faculty of Civil Engineering, Thákurova 7, 16629 Prague, Czech Republic \\ ${ }^{2}$ Institute of Information Theory and Automation of the ASCR, Pod Vodárenskou věž 4, 18208 Prague, Czech Republic
}

Corresponding author: kruzik@utia.cas.cz

\begin{abstract}
We formulate a problem of the evolution of elasto-plastic materials subjected to external loads in the framework of large deformations and multiplicative plasticity. We focus on a spontaneous inhomogenization interpreted as a structuralization process. Our model includes gradients of the plastic strain and of hardening variables which provide a relevant length scale of the model. A simple computational experiment interpreted as a hint of a deformation substructure formation is included.
\end{abstract}

Keywords: energetic solution, crystal plasticity, gradient plasticity, slip system.

\section{Introduction}

The elastic-plastic behavior of crystalline materials poses a challenge for mathematical analysis on the microscopic, the mesoscopic, and the macroscopic scales. Here, we study a rate-independent model arising in the crystal plasticity. A common and successful approach to the analysis of crystalline materials is by means of energy minimization; see e.g. Ortiz \& Repetto [30]. This is manifested for various elastic crystals, even for those with the potential of undergoing phase transitions. The applicability of variational methods has been broadened to include rate-independent evolution. Typically, these models are characterized by energy minimization of a functional including macroscopic quantities such as the macroscopic deformation gradient as well as a dissipation functional. In order to introduce a physically relevant scale to our problem we assume, following earlier works of Bažant \& Jirásek [3], Dillon \& Kratochvíl [9], Gurtin and Gurtin \& Anand [15, 16], Mainik \& Mielke [22] and others, that our energy functional depends also on the gradient of the plastic tensor. The gradient term models nonlocal effects caused by short-range interactions among dislocations. It is not clear, however, which function of the gradient should be used. For further details we refer to Kratochvíl \& Sedláček [18], and to Bakó \& Groma [1] for attempts to derive it from statistical physics revealing thus complexity of the problem, for more details. A related approach to non-local models in damage and plasticity was undertaken in Bažant \& Jirásek [3], see also [8, 10, 12, 23].

In this paper, we formulate the so-called energetic solution due to Mielke et al. [28] to our problem. This concept of a solution is based on two requirements. First, as a consequence of the conservation law for linear momentum, all work put into the system by external forces or boundary conditions is spent on increasing the stored energy or it is dissipated. Secondly, the formulation must satisfy the second law of thermodynamics, which in the present mechanical framework has the form of a dissipation inequality. The last requirement enters the framework as the assumption of the existence of a nonnegative convex potential of dissipative forces. As a consequence, the imposed deformation evolves in such a way that the sum of the stored and dissipated energies is always minimized. The main advantage of this approach is that it allows us to exploit the theory of the modern calculus of variations and suggests a numerical approach to this problem.

To expose the essence of the mathematical structure of the energetic approach, we first analyze a proto-model called here a material with internal variables. It freely follows the exposition of Francfort $\&$ Mielke [11] and we recall it here to motivate the notion of the energetic solution. In the second step, the framework is applied to elasto-plastic materials by specifications of some internal variables. One of the main results is that the described energetic approach can be identified with crystal plasticity with strain gradients in the version formulated by Gurtin [15]. Gurtin's model is formulated in the mathematical language of differential equations. From the point of view of numerical solution of a boundary value problem of crystal plasticity the energetic formulation is more convenient.

Our results are closely related to [22], where the authors proved the existence of energetic solutions to strain gradient plasticity with polyconvex energy density allowing even for $+\infty$; see [2] and to Giacomini \& Lussardi [13] where the linear-elastoplasticity 
framework is considered. Here we allow for finite quasiconvex stored energy density and large deformations. This is motivated by relaxation theory in the calculus of variation, where the effective macroscopic energy density is quasiconvexification of the microscopic energy density. Thus, our results may be applied to plasticity of materials with developing microstructures, as in shape memory alloys, [4], or [26] for instance. We refer an interested reader to [19] for a model describing cyclic plasticity in these materials. Another related paper is Carstensen et al. [6], where the authors use the energetic approach to plasticity without strain gradients.

In what follows, $\Omega \subset \mathbb{R}^{n}$, is an open bounded domain and $L^{\beta}\left(\Omega ; \mathbb{R}^{n}\right), 1 \leq \beta<+\infty$ denotes the usual Lebesgue space of mappings $\Omega \rightarrow \mathbb{R}^{n}$ whose modulus is integrable with the power $\beta$, and $L^{\infty}\left(\Omega ; \mathbb{R}^{n}\right)$ is the space of measurable and essentially bounded mappings $\Omega \rightarrow \mathbb{R}^{n}$. Further, $W^{1, \beta}\left(\Omega ; \mathbb{R}^{n}\right)$ standardly represents the space of mappings which live in $L^{\beta}\left(\Omega ; \mathbb{R}^{n}\right)$ and their gradients belong to $L^{\beta}\left(\Omega ; \mathbb{R}^{n \times n}\right)$. If $f: \mathbb{R}^{n} \rightarrow \mathbb{R}$ is convex but possibly nonsmooth we define its subdifferential at a point $x_{0} \in \mathbb{R}^{n}$ as the set of all $v \in \mathbb{R}^{n}$ such that $f(x) \geq f\left(x_{0}\right)+v \cdot\left(x-x_{0}\right)$ for all $x \in \mathbb{R}^{n}$. The subdifferential of $f$ will be denoted $\partial^{\text {sub }} f$ and its elements will be called subgradients of $f$ at $x_{0}$.

\section{Materials with internal variables}

Consider a material whose elastic properties depend on internal variables $z \in Z \subset \mathbb{R}^{m}$. The stored energy density is then $\mathcal{W}=\mathcal{W}\left(F_{\mathrm{e}}, z\right)$, where $F_{\mathrm{e}} \in \mathbb{R}^{n \times n}$ is the elastic strain. We are interested in the rateindependent evolution of the material. To this end, we assume the existence of a nonnegative convex potential $\delta=\delta(\dot{z})$ of dissipative forces, where $\dot{z}$ denotes the time derivative of $z$. In order to ensure rateindependence, $\delta$ must be positively one-homogeneous, i.e., $\delta(\alpha \dot{z})=\alpha \delta(\dot{z})$ for all $\alpha>0$. Finally, we define for $z \in Z$ a thermodynamic force

$$
Q:=-\frac{\partial}{\partial z} \mathcal{W}\left(F_{\mathrm{e}}, z\right)
$$

The evolution rule is introduced in the form

$$
Q(t) \in \partial^{\mathrm{sub}} \delta(\dot{z}(t)),
$$

where $\partial^{\text {sub }} \delta$ is the subdifferential of $\delta$. Hence, there is $\omega(t) \in \partial^{\operatorname{sub}} \delta(\dot{z}(t))$ such that $Q(t)=\omega(t)$. Maximal monotonicity of the subdifferential implies that for all $\theta \in \partial^{\text {sub }} \delta(\xi)$ we have

$$
\langle\omega(t)-\theta, \dot{z}(t)-\xi\rangle \geq 0 .
$$

Remark 2.1. In particular, taking $\xi=0$ and realizing that the one-homogeneity of $\delta$ yields $\delta(\dot{z})=\langle\omega, \dot{z}\rangle$ for all $\omega \in \partial^{\operatorname{sub}} \delta(\dot{z})$ we get

$$
\delta(\dot{z}(t))=\langle\omega(t), \dot{z}(t)\rangle=\langle Q(t), \dot{z}(t)\rangle \geq\langle\theta, \dot{z}(t))\rangle
$$

for all $\theta \in \partial^{\text {sub }} \delta(0)$. Inequality (4) expresses the socalled maximum dissipation principle (see e.g. Hill [17] or Simo [31]), which says that thermodynamic forces "available" in the so-called elastic domain $\partial^{\text {sub }} \delta(0)$ are not strong enough to overcome frictional forces.

In what follows, $\Omega \subset \mathbb{R}^{n}$, is a bounded Lipschitz domain representing the so-called reference configuration, $\nu$ is the outer unit normal to $\partial \Omega$, and $\partial \Omega \supset \Gamma_{0}, \Gamma_{1}$ which are disjoint. The deformation will be denoted $y: \Omega \rightarrow \mathbb{R}^{n}$. The evolution of the system will be controlled by external forces. Let $f(t): \Omega \rightarrow \mathbb{R}^{n}$ be the (volume) density of the external body forces and $g(t): \Gamma_{1} \subset \partial \Omega \rightarrow \mathbb{R}^{n}$ be the (surface) density of the surface forces. The equilibrium equations governing the mechanical behavior of the system are:

$$
\begin{aligned}
-\operatorname{div}\left(\frac{\partial}{\partial \nabla y} \mathcal{W}(\nabla y(t), z(t))\right) & =f(t) & & \text { in } \Omega, \\
y(t, x) & =y_{0}(x) & & \text { on } \Gamma_{0},(6) \\
\frac{\partial}{\partial \nabla y} \mathcal{W}(\nabla y(t), z(t)) \nu(x) & =g(t, x) & & \text { on } \Gamma_{1} .
\end{aligned}
$$

The full system characterizing the proto-model consists of (5)-(7) supplemented by (2):

$$
\begin{aligned}
-\frac{\partial}{\partial z} \mathcal{W}(\nabla y(t), z(t)) & \in \partial \delta(\dot{z}(t)), \\
z(0) & =z_{0}, \quad z \in Z,
\end{aligned}
$$

where $z_{0} \in Z$ is an initial condition for the internal variable.

In order to regularize our problem we may add the gradient of the internal variable, i.e., for $\omega \geq 1$ and $\varepsilon>0$ put

$$
\mathcal{W}(\nabla y, z)+\frac{\varepsilon}{\omega}|\nabla z|^{\omega} .
$$

The evolution rule changes to

$$
\begin{array}{r}
\varepsilon \operatorname{div}\left(|\nabla z(t)|^{\omega-2} \nabla z(t)\right)-\frac{\partial}{\partial z} \mathcal{W}(\nabla y(t), z(t)) \\
\in \partial \delta(\dot{z}(t)), \quad z(0)=z_{0}, \quad z \in Z
\end{array}
$$

so we have the thermodynamic force

$$
\begin{aligned}
Q(t):=\varepsilon \operatorname{div}\left(|\nabla z(t)|^{\omega-2} \nabla z(t)\right) & \\
& -\frac{\partial}{\partial z(t)} \mathcal{W}(\nabla y(t), z(t)) .
\end{aligned}
$$


The potential energy of our system can be written $(\epsilon:=\varepsilon / \omega)$

$$
\begin{aligned}
\mathcal{I}(t, y(t), z(t)) & :=\int_{\Omega} \mathcal{W}(\nabla y(t), z(t)) \mathrm{d} x \\
& +\epsilon \int_{\Omega}|\nabla z(t)|^{\omega} \mathrm{d} x-L(t, y(t)),
\end{aligned}
$$

where the work done by external forces is

$$
\begin{aligned}
L(t, y(t)):=\int_{\Omega} f(t) \cdot y(t) & \mathrm{d} x \\
& +\int_{\Gamma_{1}} g(t) \cdot y(t) \mathrm{d} S
\end{aligned}
$$

and the following energy balance is satisfied

$$
\frac{\mathrm{d}}{\mathrm{d} t} \mathcal{I}(t, y(t), z(t))=\dot{L}(t, y(t))-\frac{\mathrm{d}}{\mathrm{d} t} \operatorname{Diss}(z ;[0, t]),
$$

where

$$
\operatorname{Diss}(z ;[0, t]):=\int_{0}^{t} \int_{\Omega} \delta(\dot{z}(s)) \mathrm{d} x \mathrm{~d} s .
$$

Hence, the integration with respect to time gives

$$
\begin{aligned}
\mathcal{I}(t, y(t), z(t)) & +\operatorname{Diss}(z ;[0, t]) \\
= & \mathcal{I}(0, y(0), z(0))+\int_{0}^{t} \dot{L}(s, y(s)) \mathrm{d} s .
\end{aligned}
$$

We can also consider a more general form of $\delta$ which can also depend on $(x, z)$, i.e. $\delta:=\delta(x, z, \dot{z})$.

Typically, however, we do not have enough smoothness in the internal variable to compute the time derivative on the right-hand side of (13).

Following Mielke [24], we define the dissipation distance between two values of internal variables $z_{0}, z_{1} \in Z$ as

$$
\begin{array}{r}
D\left(x, z_{0}, z_{1}\right):=\inf _{z}\left\{\int_{0}^{1} \delta(x, z(s), \dot{z}(s)) \mathrm{d} s ;\right. \\
\left.z(0)=z_{0}, z(1)=z_{1}\right\},
\end{array}
$$

where $z \in C^{1}([0,1] ; Z)$, and set

$$
\mathcal{D}\left(z_{1}, z_{2}\right)=\int_{\Omega} D\left(x, z_{1}(x), z_{2}(x)\right) \mathrm{d} x,
$$

where $z_{1}, z_{2} \in \mathbb{Z}:=\left\{z: \Omega \rightarrow \mathbb{R}^{M} ; z(x) \in\right.$ $Z$ a.e. in $\Omega\}$. We assume that $\mathbb{Z}$ is equipped with strong and weak topologies which define the notions of convergence used below.

Following [11, 22], we impose the following assumptions on $\mathcal{D}$ :

(i) Weak lower semicontinuity:

$$
\mathcal{D}(z, \tilde{z}) \leq \liminf _{k \rightarrow \infty} \mathcal{D}\left(z_{k}, \tilde{z}_{k}\right),
$$

whenever $z_{k} \rightarrow z$ and $\tilde{z}_{k} \rightarrow \tilde{z}$. (ii) Positivity: If $\left\{z_{k}\right\} \subset \mathbb{Z}$ is bounded and at the same time $\min \left\{\mathcal{D}\left(z_{k}, z\right), \mathcal{D}\left(z, z_{k}\right)\right\} \rightarrow 0$ then

$$
z_{k} \rightarrow z \text {. }
$$

\subsection{Energetic solution}

Suppose that we look for the time evolution of $y(t) \in$ $\mathbb{Y} \subset\left\{y: \Omega \rightarrow \mathbb{R}^{n}\right\}$ and $z(t) \in \mathbb{Z}$ during the time interval $[0, T]$. The following two properties are the key ingredients of the so-called energetic solution due to Mielke and Theil [27, 28].

(i) Stability inequality: $\forall t \in[0, T], \tilde{z} \in \mathbb{Z}, y \in \mathbb{Y}$ :

$$
\mathcal{I}(t, y(t), z(t)) \leq \mathcal{I}(t, \tilde{y}, \tilde{z})+\mathcal{D}(z(t), \tilde{z})
$$

(ii) Energy balance: $\forall t, 0 \leq t \leq T$

$$
\begin{aligned}
& \mathcal{I}(t, y(t), z(t))+\operatorname{Var}(\mathcal{D}, z ;[0, t]) \\
& \quad=\mathcal{I}(s, y(0), z(0))+\int_{0}^{t} \dot{L}(\xi, y(\xi)) \mathrm{d} \xi
\end{aligned}
$$

where

$$
\begin{array}{r}
\operatorname{Var}(\mathcal{D}, z ;[s, t]):=\sup \left\{\sum_{i=1}^{N} \mathcal{D}\left(z\left(t_{i}\right), z\left(t_{i-1}\right)\right) ;\right. \\
\left.\left\{t_{i}\right\} \text { partition of }[s, t]\right\} .
\end{array}
$$

Definition 2.2. The mapping $t \mapsto(y(t), z(t)) \in \mathbb{Y} \times$ $\mathbb{Z}$ is an energetic solution to the problem $(\mathcal{I}, \delta, L)$ if the stability inequality and the energy balance are satisfied.

Remark 2.3. Note that the stability inequality (i) can be written in the form $\forall t \in[0, T], \tilde{z} \in \mathbb{Z}, \tilde{y} \in \mathbb{Y}$

$\mathcal{I}(t, y(t), z(t))+\mathcal{D}(z(t), z(t)) \leq \mathcal{I}(t, \tilde{y}, \tilde{z})+\mathcal{D}(z(t), \tilde{z})$, which means that $y(t), z(t)$ always minimizes $(\tilde{y}, \tilde{z}) \mapsto$ $\mathcal{I}(t, \tilde{y}, \tilde{z})+\mathcal{D}(z(t), \tilde{z})$. This means that the equilibrium configurations are characterized by energetic minima. Contrary to elasticity theory, the minimized energy is not only the overall elastic energy described by $\mathcal{I}$ but the dissipated energy is added.

It is convenient to put $\mathbb{Q}:=\mathbb{Y} \times \mathbb{Z}$ and to set $q:=(y, z)$. Moreover, we define the set of stable states at time $t$ as

$$
\begin{aligned}
\mathcal{S}(t):=\{q \in \mathbb{Q} ; & \forall \tilde{q} \in \mathbb{Q}: \\
& \mathcal{I}(t, q) \leq \mathcal{I}(t, \tilde{q})+\mathcal{D}(q, \tilde{q})\}
\end{aligned}
$$

and

$$
\mathcal{S}_{[0, T]}:=\bigcup_{t \in[0, T]}\{t\} \times \mathcal{S}(t) .
$$

Moreover, a sequence $\left\{\left(t_{k}, q_{k}\right)\right\}_{k \in \mathbb{N}}$ is called stable if $q_{k} \in \mathcal{S}\left(t_{k}\right)$. 


\section{Applications to elasto-plasticity}

Now we apply the energetic approach to an elastoplastic problem.

\subsection{Problem statement}

In what follows $y: \Omega \rightarrow \mathbb{R}^{n}$ will be a deformation of a body $\Omega \subset \mathbb{R}^{n}$ (in a fixed reference configuration) with the deformation gradient $F=\nabla y$. In particular, $y$ covers both elastic and plastic deformation. We define the multiplicative split, $F=F_{\mathrm{e}} F_{\mathrm{p}}$, into an elastic part $F_{\mathrm{e}}$ and an irreversible plastic part $F_{\mathrm{p}}$, which belongs to $\operatorname{SL}(n):=\left\{A \in \mathbb{R}^{n \times n} ; \operatorname{det} A=1\right\}$. The socalled plastic strain $F_{\mathrm{p}}$ and the vector $p \in \mathbb{R}^{m}$ of the hardening variables are internal variables influencing elasticity. In other words, $z(x)=\left(F_{\mathrm{p}}(x), p(x)\right) \in$ $\operatorname{SL}(n) \times \mathbb{R}^{m}$ for almost all $x \in \Omega$.

The energy functional $\mathcal{I}$ takes the form

$$
\begin{array}{r}
\mathcal{I}(t, y(t), z(t)) \\
:=\int_{\Omega} \mathcal{W}\left(x, \nabla y F_{\mathrm{p}}^{-1}, F_{\mathrm{p}}, \nabla F_{\mathrm{p}}, p, \nabla p\right) \mathrm{d} x \\
-L(t, y(t)),
\end{array}
$$

with $L$ given by (12).

In order to ease the notation, we omit the dependence of $\mathcal{W}$ on $x$. However, all the theory developed in this paper may include nonhomogeneous $\mathcal{W}$.

In what follows, we suppose that

$$
y \in \mathbb{Y}:=\left\{y \in W^{1, d}\left(\Omega ; \mathbb{R}^{n}\right) ; y=y_{0} \text { on } \Gamma_{0}\right\},
$$

where $\Gamma_{0} \subset \partial \Omega$ with a positive surface measure. Moreover, we suppose that $\Gamma_{0} \cap \Gamma_{1}=\emptyset$. Further

$$
\begin{array}{r}
\mathbb{Z}:=\left\{\left(F_{p}, p\right) \in W^{1, \beta}\left(\Omega ; \mathbb{R}^{n \times n}\right) \times W^{1, \omega}\left(\Omega ; \mathbb{R}^{m}\right) ;\right. \\
\left.F_{p}(x) \in \operatorname{SL}(n) \text { for a.e. } x \in \Omega\right\} .
\end{array}
$$

As $q=(y, z)$ it will be advantageous and will cause no confusion to write $\mathcal{D}$ as dependent on $q$, i.e.,

$$
\mathcal{D}\left(q_{1}, q_{2}\right):=\mathcal{D}\left(z_{1}, z_{2}\right)
$$

if $q_{1}=\left(y_{1}, z_{1}\right)$ and $q_{2}=\left(y_{2}, z_{2}\right)$. Similarly, we may write $\mathcal{I}$ in terms of $q=(y, z)$ as

$$
\begin{array}{r}
\mathcal{I}(t, q(t))=\int_{\Omega} \mathcal{W}\left(x, \nabla y F_{\mathrm{p}}^{-1}, F_{\mathrm{p}}, \nabla F_{\mathrm{p}}, p, \nabla p\right) \mathrm{d} x \\
-L(t, q(t)),
\end{array}
$$

where, obviously, $L(t, q(t)):=L(t, y(t))$.

In this situation, $Q=\left(Q_{1}, Q_{2}\right)$ are conjugate plastic stress and conjugate hardening forces, respectively.

$$
\begin{aligned}
Q_{1}=\operatorname{div}\left(\frac{\partial \mathcal{W}\left(\nabla y F_{\mathrm{p}}^{-1}, F_{\mathrm{p}}, \nabla F_{\mathrm{p}}, p, \nabla p\right)}{\partial \nabla F_{\mathrm{p}}}\right) \\
-\frac{\partial \mathcal{W}\left(\nabla y F_{\mathrm{p}}^{-1}, F_{\mathrm{p}}, \nabla F_{\mathrm{p}}, p, \nabla p\right)}{\partial F_{\mathrm{p}}}
\end{aligned}
$$

and

$$
\begin{aligned}
Q_{2}=\operatorname{div}( & \left.\frac{\partial \mathcal{W}\left(\nabla y F_{\mathrm{p}}^{-1}, F_{\mathrm{p}}, \nabla F_{\mathrm{p}}, p, \nabla p\right)}{\partial \nabla p}\right) \\
& -\frac{\partial \mathcal{W}\left(\nabla y F_{\mathrm{p}}^{-1}, F_{\mathrm{p}}, \nabla F_{\mathrm{p}}, p, \nabla p\right)}{\partial p} .
\end{aligned}
$$

The elastic domain is defined as

$$
\mathcal{Q}(x, z)=\partial_{\dot{z}}^{\text {sub }} \delta(x, z, 0) .
$$

Remark 3.1. The principle of maximal dissipation asserts that

$$
Q_{1}: \dot{F}_{p}+Q_{2} \cdot \dot{p}
$$

is maximal if $\dot{F}_{p}$ and $\dot{p}$ are kept fixed and $\left(Q_{1}, Q_{2}\right) \in$ $\mathcal{Q}(x, z)$. This means that for all $(A, B) \in \mathcal{Q}(x, z)$.

$$
Q_{1}: \dot{F}_{p}+Q_{2} \cdot \dot{p} \geq A: \dot{F}_{p}+B \cdot \dot{p}
$$

Finally, we conclude with an example covered by our approach.

Example 3.2 (Simple shear carried by a single slip). Consider a single slip system defined by two orthonormal vectors $a, b \in \mathbb{R}^{3}$ such that $a$ is the glide direction and $b$ is the slip-plane normal. Further suppose that we have a particular case of a so-called separable material where:

$$
\mathcal{W}\left(x, F_{\mathrm{e}}, z\right)=\mathcal{W}_{1}\left(F_{\mathrm{e}}\right)+\alpha\left|F_{\mathrm{p}}\right|^{2}+\frac{\varepsilon}{2}\left|\nabla F_{\mathrm{p}}\right|^{2},
$$

where $F_{\mathrm{p}}(t)=\mathbb{I}+\gamma(t) a \otimes b$ where $\gamma$ is the plastic slip. The slip system is generally not fixed in the reference configuration. The slip-plane normal $\tilde{b}$ in the reference configuration has the form $\tilde{b}=\left(F_{\mathrm{p}}\right)^{\top} b$. However, in this special case we have that $\tilde{b}=b$, so that the slip-plane normal is kept constant during the process [15].

Due to the special case of $F_{\mathrm{p}}$ we may identify $z:=$ $(\gamma, p)$ because $F_{\mathrm{p}}$ depends only on $\gamma$.

Choose the dissipation metric:

$$
\begin{aligned}
\delta(z, \dot{z}) & =\delta(\gamma, p, \dot{\gamma}, \dot{p}), \\
\delta(\gamma, p, \dot{\gamma}, \dot{p}) & = \begin{cases}p|\dot{\gamma}| & \text { if } \dot{p} \geq H|\dot{\gamma}|, \\
+\infty & \text { otherwise },\end{cases}
\end{aligned}
$$

where $H$ is the so-called hardening function.

The evolution rule reads:

$$
\varepsilon \Delta \gamma \in \partial^{\mathrm{sub}}(p|\dot{\gamma}|)
$$

The elastic domain $\partial^{\text {sub }} \delta(\gamma, p, 0,0)=[-p, p]$ if $\dot{p} \geq$ $H|\dot{\gamma}|$ and $(-\infty, \infty)$ otherwise. The boundary of the elastic domain $\pm p-\varepsilon \Delta \gamma=0$ defines the yield surface. Thus, the energetic approach recovers Gurtin's calculations on shear bands in single-slip, see Gurtin (2000). 
The dissipation distance is:

$$
\begin{aligned}
& \mathcal{D}\left(\gamma_{1}, p_{1}, \gamma_{2}, p_{2}\right) \\
& \quad= \begin{cases}p_{2}\left|\gamma_{2}-\gamma_{1}\right| & \text { if } p_{2}-p_{1} \geq H\left|\gamma_{2}-\gamma_{1}\right|, \\
+\infty & \text { otherwise }\end{cases}
\end{aligned}
$$

If $H>0$ we get that the optimal choice is $p_{2}=$ $p_{1}+H\left|\gamma_{2}-\gamma_{1}\right|$ which gives $\mathcal{D}\left(\gamma_{1}, p_{1}, \gamma_{2}, p_{2}\right)=p_{1} \mid \gamma_{2}-$ $\gamma_{1} \mid+H\left(\gamma_{2}-\gamma_{1}\right)^{2}$.

The following finite element computation illustrates the performance of our model. We take $n=2$, $\Omega=(0,1) \times(0,3), t \in[0,1]$. Further, we apply a zero Dirichlet boundary condition on $(0,1) \times\{0\}$ and $u(t, x)=-0.6 t$ for $x=(0,1) \times\{3\}$. As to material properties we consider a homogeneous isotropic material with a Young modulus $200 \mathrm{GPa}$, Poisson's ratio $0.3, a=(-1,1) / \sqrt{2}, b=(1,1) / \sqrt{2}$ and the $\mathcal{W}_{1}(E)=\frac{\lambda}{2} \operatorname{tr}|E|^{2}+\mu|E|^{2}$ where $\lambda$ and $\mu$ are Lamé constants corresponding to the used Young modulus and to the Poisson's ratio. The other model constants were set as follows: $\varepsilon=40 \mathrm{GPa} \cdot \mathrm{m}^{2}, \alpha=2 \mathrm{MPa}$, and $H=0.2 \mathrm{MPa}$.

The initial condition was chosen $\gamma=0$ (no plastic deformation) and initial yield stress $p=200 \mathrm{MPa}$. Note that the constant defining the energy stored in defects, i.e., $\alpha$, is much smaller than the elastic constants of the material. The specimen was discretized using piecewise affine finite elements for the displacement as well as for $F_{\mathrm{p}}$. A sequence of resulting minimization problems was solved by a Fortran 77 routine described in [5], which is based on a quasiNewton method. It is designed to solve large-scale nonlinear optimization problems with box constraints. We first observe the development of 45-degree bands in the vicinity of the boundary where the Dirichlet boundary conditions are applied. At the final stage a large plastic deformation appears in the middle of the specimen (see e.g. [20] for similar calculations). The spontaneously-formed inhomogeneity provides a hint of a deformation structuralization process. The characteristic length scale introduced through the higher gradients guarantees the intrinsic size of the inhomogeneity independent of the FEM mesh size.

\section{Acknowledgements}

This work was supported by GAČR through projects P107/12/0121, P201/10/0357, and by the research project VZ-MŠMT 6840770003.

\section{References}

[1] Bakó, B., Groma, I.: Stochastic approach for modeling dislocation patterning, Phys. Rev. B., 60, 1999, 122-127.
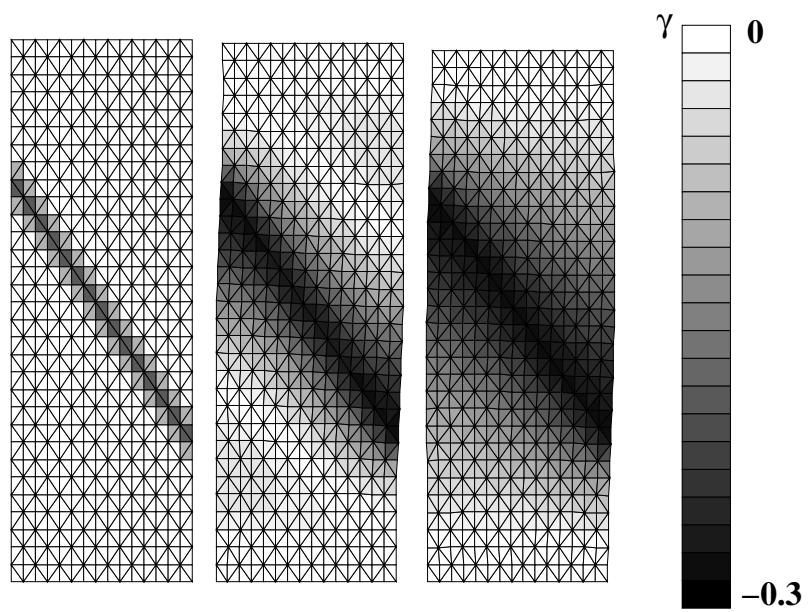

Figure 1: Elasto-plastic deformation of a twodimensional specimen at four time instants. The darker the shade of gray color, the larger is the plastic deformation, i.e., $|\gamma|$. The loading increases from left to right.

[2] Ball, J.M.: Convexity conditions and existence theorems in nonlinear elasticity, Arch. Ration. Mech. Anal., 63, 1997, 337-403.

[3] Bažant, Z.P., Jirásek, M.: Nonlocal integral formulation of plasticity and damage: a survey of progress, J. Engrg. Mech., 128, 2002, 11191149 .

[4] Bhattacharya, K.: Microstructure of Martensite. Why it forms and how it gives rise to the shapememory effect. Oxford: Oxford University Press, 2003.

[5] Byrd, R.H., Lu, P., Nocedal, J., Zhu, C.: A limited memory algorithm for bound constrained optimization problems, SIAM J. Scientific Computing, 16, 1995, 1190-1208.

[6] Carstensen, C., Hackl, K. Mielke, A.: Nonconvex potentials and microstructures in finite-strain plasticity, Proc. Roy. Soc. Lond. A, 458, 2002, 299-317.

[7] Dacorogna, B.: Direct Methods in the Calculus of Variations 2nd ed. New York: Springer, 2008.

[8] Dal Maso, G., Francfort, G., Toader, R.: A model of quasistatic crack growth of brittle fractures: existence and approximation results, Arch. Ration. Mech. Anal., 176, 2005, 165-225.

[9] Dillon, O.W., Kratochvíl, J.: A strain gradient theory of plasticity, Int. J. Solid Struct., 6, 1970, 1513-1533.

[10] Fleck, N., Hutchinson, J.W.: A phenomenological theory for strain gradient effects in plasticity. J. Mech. Phys. Solids, 41, 1993, 1825-1857. 
[11] Francfort, G., Mielke, A.: Existence results for a class of rate-independent material models with nonconvex elastic energies, J. reine angew. Math., 595, 2006, 55-91.

[12] Frémond, M.: Non-Smooth Thermomechanics. Berlin: Springer, 2002.

[13] Giacomini, A., Lusardi L.: Quasistatic evolution for a model in strain gradient plasticity, SIAM J. Math. Anal., 40, 2008, 1201-1245.

[14] Groma, I.: Link between the microscopic and mesoscopic length-scale description of the collective behaviour of dislocations, Phys. Rev. B, 56, $1997,5807$.

[15] Gurtin, M.E.: On the plasticity of single crystals: free energy, microforces, plastic-strain gradients, J. Mech. Phys. Solids, 48, 2000, 989-1036.

[16] Gurtin, M.E., Anand, L.: A theory of straingradient plasticity for isotropic, plastically irrotational materials. I. Small deformations, J. Mech. Phys. Solids, 53, 2005, 1624-1649.

[17] Hill, R.: A variational principle of maximum plastic work in classical plasticity, Q. J. Mech. Appl. Math., 1, 1948, 18-28.

[18] Kratochvíl, J., Sedláček, R.: Statistical foundation of continuum dislocation plasticity, Phys. Rev. B 77, 2008, 134102.

[19] Kružík, M., Zimmer, J.: A model of shape memory alloys accounting for plasticity, IMA J. Appl. Math., 76, 2010, 193-216.

[20] Kuroda, M., Tvergaard, V.: A finite deformation theory of higher-order gradient crystal plasticity, J. Mech. Phys. Solids, 56, 2008, 2573-2585.

[21] Mainik, A., Mielke, A.: Existence results for energetic models for rate-independent systems. Calc. Var. Partial Differential Equations, 22, 2005, 73-99.

[22] Mainik, A., Mielke, A.: Global existence for rateindependent gradient plasticity at finite strain, J. Nonlinear Sci., 19, 2009, 221-248.
[23] Maugin, G.A.: The Thermomechanics of Plasticity and Fracture Cambridge : University Press, 1992.

[24] Mielke, A.: Energetic formulation of multiplicative elasto-plasticity using dissipation distances. Cont. Mech. Thermodyn., 15, 2002, 351-382.

[25] Mielke, A.: Evolution of rate-independent systems. In: Evolutionary equations. II, Handb. Differ. Equ., pp. 461-559, Amsterdam :Elsevier/North-Holland, 2005.

[26] Mielke, A., Roubíček, T.: A rate-independent model for inelastic behavior of shape-memory alloys, Multiscale Model. Simul., 1, 2003, 571597.

[27] Mielke, A., Theil, F.: A mathematical model for rate-independent phase transformations with hysteresis. In: Models of continuum mechanics in analysis and engineering. (Eds.: H.-D.Alder, R.Balean, R.Farwig), Aachen :Shaker Verlag, 1999, pp.117-129.

[28] Mielke, A., Theil, F., Levitas, V.I.: A variational formulation of rate-independent phase transformations using an extremum principle, Arch. Ration. Mech. Anal. 162, 2002, 137-177.

[29] Mühlhaus, H-B., Aifantis, E.C.: A variational principle for gradient plasticity, Int. J. Solid. Structures 28, 1991, 845-857.

[30] Ortiz, M., Repetto, E.A.: Nonconvex energy minimization and dislocation structures in ductile single crystals. J. Mech. Phys. Solids, 47, 1999, 397-462.

[31] Simo, J.: A framework for finite strain elastoplasticity based on maximum plastic dissipation and multiplicative decomposition. Part I. Continuum formulation, Comp. Meth. Appl. Mech. Engrg., 66, 1988, 199-219. Part II. Computational Aspects, Comp. Meth. Appl. Mech. Engrg. 68, 1988, $1-31$.

[32] Tsagrakis, I., Aifantis, E.C.: Recent developments in gradient plasticity. J. Engrg. Mater. Tech. 124, 2002, 352-357. 